\title{
A constructive condition for dynamic feedback linearization
}

\author{
Stefano Battilotti, Claudia Califano* \\ Dipartimento di Informatica e Sistemistica "Antonio Ruberti”, Università di Roma "La Sapienza”, Via Eudossiana 18, \\ 00184 Roma, Italy
}

Received 28 August 2002; received in revised form 26 October 2003; accepted 10 February 2004

\begin{abstract}
The paper deals with dynamic feedback linearization of continuous time affine systems. A constructive procedure based on prolongations is proposed. Based on necessary geometric conditions, the algorithm computes a set of prolongation indices. (c) 2004 Elsevier B.V. All rights reserved.
\end{abstract}

Keywords: Dynamic feedback linearization; Prolongations; Differential geometry

\section{Introduction}

The feedback linearization problem has been widely studied both in continuous and in discrete time (see $[11,20]$ and the references therein). The first works deal with the exact linearization problem $[4,5,10,12,14-18]$, while dynamic solutions were considered in $[6,13,19]$. In [7], sufficient geometric conditions via prolongations and diffeomorphism were given. The sufficiency concerns two aspects: the a priori knowledge of a candidate set of prolongation indices and the restriction to solutions which guarantee a projective relation between the extended system and the original one. The equivalence of differentially flat systems to dynamic feedback linearizable systems was instead considered in $[8,9]$ where the concept of endogenous feedback was introduced: the solution is in this case based on the knowledge of a set of flat outputs, i.e. linearizing output functions depending on the state, the control and its derivatives. Algebraic

\footnotetext{
${ }^{*}$ Corresponding author.

E-mail address: claudia.califano@uniroma1.it (C. Califano).
}

necessary and sufficient conditions under endogenous feedback, were given in $[1,2]$. A bound on the number of integrators necessary to achieve linearization was given in [21].

In the present work we propose an algorithm for the computation of a dynamic solution consisting of prolongations and based on a set of necessary geometric conditions which become sufficient when the prolongation indices are at most equal to 2 . The basic idea beyond the algorithm is that the loss of involutivity and/or constant dimensionality of the distributions $\mathscr{G}_{i}:=\operatorname{span}\left\{g \cdots a d_{f}^{i} g\right\}$, which characterize the static feedback solution, is linked to appropriate directions which must be added to the $\mathscr{G}_{i}$ 's. This corresponds to identify those inputs which should not be extended through prolongations. Furthermore, the geometric interpretation in terms of directions immediately clarifies the use of more general feedback laws. In fact, the above-mentioned directions may not coincide, in general, with the directions of the $a d_{f}^{i+1} g_{j}$ 's, but rather with linear combinations of them. They can then be obtained by recombining the inputs via a static feedback, so that the algorithm may provide linearizing 
feedback laws more general than prolongations. This generalization would require, at each step, the computation of the new closed-loop vector fields. To avoid this computational burdening, we have restricted ourselves to the class of dynamic feedback laws composed of prolongations plus a final static feedback on the extended system. The procedure is illustrated on a dynamic feedback linearizable system used in [7] to show that it did not satisfy the proposed sufficient conditions. The proposed algorithm computes the desired prolongation indices. Preliminary results were proposed in [3].

Statement of the problem. Consider the continuous time analytic system

$\dot{x}=f(x)+\sum_{i=1}^{m} g_{i}(x) u_{i}$,

where $x \in \mathbb{R}^{n}$, and $f(x), g_{1}(x), \ldots, g_{m}(x)$ are analytic defined on a open set of $\mathbb{R}^{n}$. Find, if there exists, a regular dynamic feedback

$u_{j}=\zeta_{j, 1}, \quad \dot{\zeta}_{j}=A_{j} \zeta_{j}+B_{j} v_{j}$,

$A_{j}=\left(\begin{array}{ll}0 & I \\ 0 & 0\end{array}\right), \quad B_{j}=\left(\begin{array}{l}0 \\ 1\end{array}\right), \quad j=1, \ldots, m$

with $A_{j}$ and $B_{j}$ of dimension $\mu_{j} \times \mu_{j}$ and $\mu_{j} \times 1$, respectively, $\zeta_{j}=\left(\zeta_{j, 1}, \ldots, \zeta_{j, \mu_{j}}\right)^{\mathrm{T}}, \zeta=\left(\zeta_{1}^{\mathrm{T}}, \ldots, \zeta_{m}^{\mathrm{T}}\right)^{\mathrm{T}}$, and such that the extended system

$$
\left(\begin{array}{c}
\dot{x} \\
\dot{\zeta}
\end{array}\right)=F(x, \zeta)+\sum_{i=1}^{m} G_{i}(x, \zeta) v_{i}
$$

is static feedback equivalent to a linear system, i.e. there exists a regular static state feedback $v=\alpha(x, \zeta)+$ $\beta(x, \zeta) w$, such that the closed-loop system is diffeomorphic to a linear system. In (2) if $u_{j}=v_{j}$ we set $\mu_{j}=0$.

We recall that the indices $\mu_{i}$ are the prolongation indices and (1) will be said dynamic feedback linearizable with prolongation indices $\left(\mu_{1} \cdots \mu_{m}\right)$. If (1) is dynamic feedback linearizable with prolongations only, then at least one prolongation index can be set to zero, i.e. $0=\mu_{1} \leqslant \cdots \leqslant \mu_{m}[21]$.

\section{Preliminaries}

The following notation will be used. Given a number $p,\lfloor p\rfloor$ will denote its inferior integer and $p !:=$ $p(p-1) \cdots 1$ the factorial number. Given two smooth vector fields $f$ and $g_{i}, a d_{f} g_{i}:=\left[f, g_{i}\right]=\left(\partial g_{i} / \partial x\right) f-$ $(\partial f / \partial x) g_{i}$, is the standard Lie bracket of vector fields, and $a d_{f}^{k} g_{i}=a d_{f}\left(a d_{f}^{k-1} g_{i}\right) ; \sum_{i=1}^{2} f_{i} \partial / \partial x_{i}$ denotes the vector field $\left(f_{1}^{\mathrm{T}}, f_{2}^{\mathrm{T}}\right)^{\mathrm{T}}$ in the coordinates $\left(x_{1}^{\mathrm{T}}, x_{2}^{\mathrm{T}}\right)^{\mathrm{T}}$. We will denote by $g=\left(g_{1} \cdots g_{m}\right)$, by $G=\left(G_{1} \cdots G_{m}\right)$, by $\mathscr{G}_{i}$ the distribution $\mathscr{G}_{i}:=\operatorname{span}\left\{g, \ldots, a d_{f}^{i} g\right\}$, by $\overline{\mathscr{G}}_{i}$, the involutive closure of $\mathscr{G}_{i}$. The $\mathscr{G}_{i}$ 's play a crucial role in the solution of the regular static feedback linearization problem. We recall the following result.

Theorem 1 (Hunt et al. [10]). Suppose that the matrix $g\left(x_{0}\right)$ has rank $m$. Then the state space exact linearization problem is solvable if and only if

(i) for each $0 \leqslant i \leqslant n-1$, the distribution $\mathscr{G}_{i}$ has constant dimension near $x_{0}$,

(ii) the distribution $\mathscr{G}_{n-1}$ has dimension $n$,

(iii) for each $0 \leqslant i \leqslant n-2$, the distribution $\mathscr{G}_{i}$ is involutive.

In [7] instead, it was shown that if, after a possible reordering of the inputs, there exists a set of integers $0=l_{1} \leqslant \cdots \leqslant l_{m}$, such that the distributions $\Delta_{0}=\operatorname{span}\left\{g_{j}: l_{j}=0\right\}, \quad \Delta_{i+1}=\Delta_{i}+$ $a d_{f} \Delta_{i}+\operatorname{span}\left\{g_{j}: l_{j}=i+1\right\} i \geqslant 0$ satisfy appropriate properties, then the problem is solvable via prolongations.

Theorem 2 (Charlet et al. [7]). If locally around $x_{0}$ there exists $0=l_{1} \leqslant \cdots \leqslant l_{m}$, such that

(i) $\Delta_{i}$ is involutive and of constant dimension for $0 \leqslant i \leqslant n+l_{m}-1$,

(ii) $\operatorname{dim}\left(\Delta_{n+l_{m}-1}\right)=n$,

(iii) $\left[g_{s}, \Delta_{i}\right] \subset \Delta_{i+1}, \forall s$ such that $l_{s} \geqslant 1$ and $\forall i$, $0 \leqslant i \leqslant n+l_{m}-1$,

then (1) is locally dynamic feedback linearizable with prolongation indices $\mu_{1} \cdots \mu_{m}$, with $\mu_{i}=l_{i}, i=1, \ldots, m$.

The sufficiency of the previous conditions stands essentially in condition (iii). In fact let us first note that since $l_{i}=\mu_{i}, i=1, \ldots, m$, for $l=0 \ldots, n+\mu_{m}-1$,

$$
\begin{gathered}
\Delta_{l}=\operatorname{span}\left\{g_{i}, \ldots, a d_{f}^{l-\mu_{i}} g_{i}, i=1, \ldots, j-1,\right. \\
\left.j: \mu_{j}>l \geqslant \mu_{j-1}, j \geqslant 2\right\} .
\end{gathered}
$$


Consider now the extended system (3) and let $\mathscr{G}_{i}^{e}:=$ $\operatorname{span}\left\{G, a d_{F} G, \ldots, a d_{F}^{i} G\right\}$. Since (3) is given by (1)(2), (iii) implies that the $a d_{F}^{s} G_{j}$ 's have the form

$A d_{F}^{S} G_{j}$

$=\left(\sum_{i=0}^{s-\mu_{1}} \eta_{i 1}(\zeta) a d_{f}^{i} g_{1}+\cdots+\sum_{i=0}^{s-\mu_{m}} \eta_{i m}(\zeta) a d_{f}^{i} g_{m}\right) \frac{\partial}{\partial x}$

$+\eta(x, \zeta) \frac{\partial}{\partial \zeta}$

which is a particular case since the projection of $\mathscr{G}_{i}^{e}$ onto $\mathbb{R}^{n}$ is given by $\Delta_{i}$. Moreover, Theorem 2 is based on the a priori knowledge of the prolongation indices $\mu_{1}, \ldots, \mu_{m}$. With respect to this result we will propose an algorithm which allows the following improvements:

- A constructive computation of a set of prolongation indices with respect to which the distributions $\Delta_{i}$ satisfy conditions (i) and (ii) of Theorem 2.

- The computation of solutions which do not necessarily satisfy condition (iii), so that the projection of $\mathscr{G}_{i}^{e}$ may not coincide with $\Delta_{i}$.

\subsection{The properties of the extended system}

Consider the extended dynamics (1)-(2), and assume it is static feedback linearizable. After a possible reordering of the inputs, $0=\mu_{1} \leqslant \cdots \leqslant \mu_{m}$. Let $s$ be the smallest index s.t. $\mu_{s} \geqslant 1$; then the closed-loop system is given by

$\dot{x}=f(x)+\sum_{i=1}^{s-1} g_{1}(x) v_{i}+\sum_{i=s}^{m} g_{i}(x) \zeta_{i 1}$

$\dot{\zeta}_{j}=A_{j} \zeta_{j}+B_{j} v_{j}, \quad j=s, \ldots, m$.

The extended system (3) is thus characterized by the following vector fields:

$$
\begin{aligned}
& F=\left(f(x)+\sum_{j=s}^{m} g_{j}(x) \zeta_{j 1}\right) \frac{\partial}{\partial x}+\sum_{j=s}^{m} A_{j} \zeta_{j} \frac{\partial}{\partial \zeta_{j}}, \\
& G_{i}= \begin{cases}g_{i}(x) \frac{\partial}{\partial x}, & i \in[1, s-1], \\
B_{j} \frac{\partial}{\partial \zeta_{j}}, & j \in[s, m] .\end{cases}
\end{aligned}
$$

As a consequence, the generic term $a d_{F}^{l+\mu_{i}} G_{i}$, (with $\left.l+\mu_{i} \geqslant 0\right)$ has the form

$$
a d_{F}^{l+\mu_{i}} G_{i}=(-1)^{\mu_{i}}\left(\tau_{i}^{l}(x, \zeta) \frac{\partial}{\partial x}+(-1)^{l} A_{i}^{l+\mu_{i}} B_{i} \frac{\partial}{\partial \zeta_{i}}\right) .
$$

In (6), for $-\mu_{i} \leqslant l<0, \tau_{i}^{l}=0$, and $A_{i}^{l+\mu_{i}} B_{i}=e_{l}$ the canonical vector with the $l$ th element equal to one and the others all zero; for $l \geqslant 0, A_{i}^{l+\mu_{i}} B_{i}=0$ and

$$
\begin{aligned}
\tau_{i}^{l}(x, \zeta):= & a d_{f}^{l} g_{i} \\
& +\sum_{j=s}^{m} \sum_{k=1}^{\min \left(\mu_{j}, l\right)} \sum_{r=0}^{l-k} c_{i r}^{k} a d_{f}^{r}\left[g_{j}, a d_{f}^{l-k-r} g_{i}\right] \zeta_{j k} \\
& +\mathcal{O}\left(\|\zeta\|^{2}\right),
\end{aligned}
$$

where

$c_{i r}^{j}=\frac{(r+j-1) !}{r !(j-1) !}$

and $\mathcal{O}\left(\|\zeta\|^{2}\right)$ represents terms of order greater than one in $\zeta$. Let $\bar{s}$ be the smallest integer such that $\mu_{\bar{s}} \geqslant 2$; for the first terms one gets

$$
\begin{aligned}
\tau_{i}^{0}(x, \zeta)= & g_{i}(x), \\
\tau_{i}^{1}(x, \zeta)= & a d_{f(x)} g_{i}(x)+\sum_{j=s}^{m} c_{i 0}^{1} a d_{g_{j}(x)} g_{i}(x) \zeta_{j 1}, \\
\tau_{i}^{2}(x, \zeta)= & a d_{f}^{2} g_{i}(x) \\
& +\sum_{j=s}^{m} \sum_{r=0}^{1} c_{i r}^{1} a d_{f(x)}^{r}\left[g_{j}(x), a d_{f(x)}^{1-r} g_{i}(x)\right] \zeta_{j 1} \\
& +\sum_{j=\bar{s}}^{m} c_{i 0}^{1} a d_{g_{j}(x)} g_{i}(x) \zeta_{j 2}+\mathcal{O}\left(\|\zeta\|^{2}\right) .
\end{aligned}
$$

The distributions $\mathscr{G}_{i}^{e}$ associated with the extended system (1)-(2), are then

$$
\begin{aligned}
\mathscr{G}_{0}^{e}= & \operatorname{span}\left\{g_{1} \frac{\partial}{\partial x}, \ldots, g_{s-1} \frac{\partial}{\partial x}\right\} \\
& +\operatorname{span}\left\{\frac{\partial}{\partial \zeta_{s, \mu_{s}}}, \ldots, \frac{\partial}{\partial \zeta_{m, \mu_{m}}}\right\} \\
\mathscr{G}_{i}^{e}= & \mathscr{G}_{i-1}^{e}+\operatorname{span}\left\{\tau_{l}^{\left(i-\mu_{l}\right)} \frac{\partial}{\partial x}, l=1, \ldots, j\right\} \\
& +\operatorname{span}\left\{\frac{\partial}{\partial \zeta_{l, \mu_{l}-i}}, l=j+1, \ldots, m\right\} i>0,
\end{aligned}
$$


where the index $j \in[1, m]$, is such that $\mu_{j} \leqslant i<\mu_{j+1}$, and by definition $\mu_{m+1}=+\infty$. Since the extended system is linearizable via regular static feedback, the distributions $\mathscr{G}_{i}^{e}$ must be involutive and regular (Theorem 1). This fact induces certain properties on the Lie brackets of the vector fields defined on the original system as stated below and proven in the Appendix.

Proposition 1. Suppose that (1) is dynamic feedback linearizable with prolongation indices $0=$ $\mu_{1} \leqslant \cdots \leqslant \mu_{m}$ around $\left(x_{0}, \zeta_{0}\right)=\left(x_{0}, 0\right)$, and consider the distribution $\Delta_{s}$ defined in (4). Locally around $x_{0}$, the following properties hold:

(a) $\Delta_{s}$ has constant dimension and is involutive, for $0 \leqslant s \leqslant n+\mu_{m}-1$

(b) $\operatorname{dim} \Delta_{n+\mu_{m}-1}=n$,

(c) for any two indices $(t, l)$ such that $\mu_{t} \leqslant s$ and $t<l$,

$$
\begin{aligned}
& {\left[a d_{f}^{r_{1}} g_{l}, a d_{f}^{r_{2}} g_{t}\right] \in \Delta_{s}, \quad \forall r_{1}, r_{2} \geqslant 0:} \\
& \quad r_{1}+r_{2}=s-\mu_{l}+1, \ldots, s-\mu_{l}+\left\lfloor\frac{\mu_{l}-\mu_{t}}{2}\right\rfloor .
\end{aligned}
$$

Moreover, if $\mu_{m} \leqslant 2$ the conditions are also sufficient.

Theorem 2 states that if the system is controllable, the distributions $\Delta_{s}$ are involutive, which are necessary conditions, and additionally $\left[g_{j}, \Delta_{s}\right] \subset \Delta_{s+1}$, then the problem is solvable. This last condition corresponds to require that

$\left[\frac{\partial}{\partial \zeta_{l, k}}, \tau_{t}^{i}(x, \zeta) \frac{\partial}{\partial x}\right]=\frac{\partial\left(\tau_{t}^{i}(x, \zeta)\right)}{\partial \zeta_{l, k}} \frac{\partial}{\partial x} \in \mathscr{G}_{s}^{e}$

for $k=1, \ldots, \mu_{l}$.

As opposite to (c) in Proposition 1, this condition must be satisfied even though $\partial / \partial \zeta_{l, k} \notin \mathscr{G}_{s}^{e}$.

\section{Main result}

Hereafter we propose an algorithm to compute a set of prolongation indices $\mu_{1} \leqslant \cdots \leqslant \mu_{m}$ w.r.t. which the associated distributions $\Delta_{i}$ satisfy the necessary conditions of Proposition 1. At the generic step $s$ we will denote by $\mu_{i}^{s}$, the prolongation index $\mu_{i}$ and by $\Delta_{i}^{s}$ the distribution $\Delta_{i}$.

The algorithm starts with $\mu_{1}^{0}=\cdots=\mu_{m}^{0}=0$ and $k_{0}=k: \operatorname{dim}\left(\Delta_{k}^{0}\right)=n$ while $\operatorname{dim}\left(\Delta_{k-1}^{0}\right)<n$. The generic step $s+1$ starts with $\mu_{1}^{s} \leqslant \cdots \leqslant \mu_{m}^{s}$ and a given $k_{s}$ for which Proposition 1 is satisfied by the distributions $\Delta_{j}^{s}, j \geqslant k_{s}$. The algorithm checks if it is satisfied for $j=k_{s}-1$ also. If not, the prolongation indices are modified accordingly. This is done in two phases. Phase 1 concerns condition (a) of Proposition 1: if $\Delta_{k_{s}-1}^{s}$ is not involutive, the algorithm computes its involutive closure which is contained in $\Delta_{k_{s}}^{s}$. By construction

$$
\begin{gathered}
\Delta_{k_{s}}^{s}=\Delta_{k_{s}-1}^{s}+\operatorname{span}\left\{a d_{f}^{k_{s}-\mu_{1}^{s}} g_{1} \cdots a d_{f}^{k_{s}-\mu_{l}^{s}} g_{l},\right. \\
\left.l: \mu_{l}^{s} \leqslant k_{s}<\mu_{l+1}^{s}\right\} .
\end{gathered}
$$

The algorithm adds to $\Delta_{k_{s}-1}^{s}$ the minimum number $j$ of elements $a d_{f}^{k_{s}-\mu_{i}^{s}} g_{i}$, such that the obtained distribution $\Delta_{k_{s}-1}^{s}+\operatorname{span}\left\{a d_{f}^{k_{s}-\mu_{l_{1}}^{s}} g_{l_{1}} \cdots a d_{f}^{k_{s}-\mu_{l_{j}}^{s}} g_{l_{j}}\right\} \supseteq \bar{\Delta}_{k_{s}-1}^{s}$

and has constant dimension locally around $x_{0}$; since the involutivity may not be preserved, the algorithm computes the smallest integer $\bar{k}_{s} \geqslant k_{s}$ such that

$$
\begin{aligned}
\bar{\Delta}_{\bar{k}_{s}-1}^{s} & \equiv \Delta_{\bar{k}_{s}-1}^{s}+\operatorname{span}\left\{a d_{f}^{\overline{k_{s}}-\mu_{l_{1}}^{s}} g_{l_{1}} \cdots a d_{f}^{\bar{k}_{s}-\mu_{l_{j}}^{s}} g_{l_{j}}\right\} \\
& :=\Delta_{k_{s+1}}^{s+1} .
\end{aligned}
$$

The prolongation indices are updated accordingly.

Phase 2 concerns instead condition (c) of Proposition 1. The algorithm checks the condition and adds the minimum number $\bar{\gamma} \geqslant 0$ of elements of the form $a d_{f}^{\bar{k}_{s}-\mu_{l_{i}}^{s}} g_{l_{i}}$ in order to satisfy the cited condition. This operation changes the prolongation indices which are updated accordingly. As a consequence the operation must be iterated on the new obtained distribution until condition (c) is satisfied by the final computed distribution $\tilde{\Delta}_{\tilde{k}_{s+1}}$. The previous operation may in general not preserve the involutivity for the new distributions, so that the algorithm computes the first index $r$ such that $\tilde{\Delta}_{\tilde{k}_{s+1}+r} \equiv \Delta_{k_{s+1}+r}$ and has constant dimension locally around $x_{0}$. Finally, a flag is introduced which points out whether checking condition (c) on $\Delta_{k_{s+1}}$ ensures that it is satisfied on $\Delta_{k_{s+1}+i}$, for any $i \geqslant 0$ or not. In this case $k_{s+1}$ is modified appropriately. 
Table 1

The left-hand side indicates the integrators to be added on each input channel. At each step an integrator is added/eliminated s.t. each row has the same number of elements of the first row

\begin{tabular}{|c|c|c|c|c|c|c|c|}
\hline & & & $g_{1}$ & $\ldots$ & $a d_{f}^{k_{s}-\mu_{m}^{s}} g_{1}$ & $\ldots$ & $a d_{f}^{k_{s}} g_{1}$ \\
\hline & & & $\vdots$ & $\vdots$ & : & $\vdots$ & $\ldots$ \\
\hline & & & $\vdots$ & . & : & $\ldots$ & $a d_{f}^{k_{s}} g_{i_{s, \mu_{m}^{s}}}$ \\
\hline & & : & $\vdots$ & $\ldots$ & $\vdots$ & $\therefore$ & \\
\hline$\frac{\partial}{\partial \xi_{m, \mu_{m}^{s-1}}^{s-1}}$ & $\ldots$ & $\frac{\partial}{\partial \xi_{m, 1}}$ & $g_{m}$ & $\ldots$ & $a d_{f}^{k_{s}-\mu_{m}^{s}} g_{m}$ & & \\
\hline
\end{tabular}

\subsection{The algorithm}

Suppose that the system is locally controllable around $u=0$, i.e. $\operatorname{dim} \mathscr{G}_{n-1}=n$, and let $k$ be the first integer such that $\operatorname{dim} \mathscr{G}_{k}=n$ and $\operatorname{dim} \mathscr{G}_{k-1}<n$.

Step 0: Set $\mu_{1}^{0}=\cdots=\mu_{m}^{0}=0, k_{0}=k$ and consider $\Delta_{k_{0}}^{0}=\operatorname{span}\left\{g, \ldots, a d_{f}^{k_{0}} g\right\}$. By construction $\Delta_{k_{0}}^{0}$ satisfies Proposition 1 since it has constant dimension $n$.

Step $s+1$ : Let $0=\mu_{1}^{s} \leqslant \cdots \leqslant \mu_{m}^{s}$ be the prolongation indices computed at Step $s$ and let the corresponding distribution (represented in Table 1) be

$\Delta_{k_{s}}^{s}=\Delta_{k_{s}-\mu_{m}^{s}}^{0}$

$$
+\sum_{j=1}^{\mu_{m}^{s}} \operatorname{span}\left\{a d_{f}^{k_{s}-\mu_{m}^{s}+j} g_{1}, \ldots, a d_{f}^{k_{s}-\mu_{m}^{s}+j} g_{i_{s, j}}\right\} .
$$

Phase 1. Consider now $\Delta_{k_{s}-1}^{s}$ which satisfies $\Delta_{k_{s}}^{s}=\Delta_{k_{s}-1}^{s}+\operatorname{span}\left\{a d_{f}^{k_{s}-\mu_{1}^{s}} g_{1}, \ldots, a d_{f}^{k_{s}-\mu_{l}^{s}} g_{l}\right.$,

$$
\left.l: \mu_{l}^{s} \leqslant k_{s}<\mu_{l+1}^{s}\right\} \text {. }
$$

Let $\bar{\Delta}_{k_{\mathrm{s}}-1}^{s}$ be the involutive closure of $\Delta_{k_{\mathrm{s}}-1}^{s}$ and set $p_{s+1}=\operatorname{dim} \bar{\Delta}_{k_{s}-1}^{s}-\operatorname{dim} \Delta_{k_{s}-1}^{s}$. Then there exist $p_{s+1}$ vector fields, $\sigma_{1}, \ldots, \sigma_{p_{s+1}}$, linear combination of elements of $\Delta_{k_{s}}^{s}$, s.t. $\bar{\Delta}_{k_{s}-1}^{s}=\Delta_{k_{s}-1}^{s}+$ $\operatorname{span}\left\{\sigma_{1} \cdots \sigma_{p_{s+1}}\right\} \subseteq \Delta_{k_{s}}^{s}$.

Let $j$, with $l \geqslant j \geqslant p_{s+1}$, and $r \geqslant 0$, be the smallest integers such that $\Delta_{k_{s}-1}^{s}+\operatorname{span}\left\{a d_{f}^{k_{s}-\mu_{l_{1}}^{s}} g_{l_{1}} \ldots\right.$ $\left.a d_{f}^{k_{s}-\mu_{l_{j}}^{s}} g_{l_{j}}\right\}$ has constant dimension $\operatorname{dim}\left(\Delta_{k_{s}-1}^{s}\right)+j$ locally around $x_{0}$ and

$$
\begin{aligned}
\bar{\Delta}_{k_{s}-1}^{s} \subseteq & \Delta_{k_{s}-1}^{s}+\operatorname{span}\left\{a d_{f}^{k_{s}-\mu_{l_{1}}^{s}} g_{l_{1}} \cdots a d_{f}^{k_{s}-\mu_{l_{j}}^{s}} g_{l_{j}}\right\}, \\
\bar{\Delta}_{k_{s}-1+r}^{s} \equiv & \Delta_{k_{s}-1+r}^{s} \\
& \quad+\operatorname{span}\left\{a d_{f}^{k_{s}-\mu_{l_{1}}^{s}+r} g_{l_{1}} \cdots a d_{f}^{k_{s}-\mu_{l_{j}}^{s}+r} g_{l_{j}}\right\} .
\end{aligned}
$$

Set $\bar{k}_{s}=k_{s}+r$ and

$\Delta_{k_{s+1}}^{s+1}:=\Delta_{\bar{k}_{s}-1}^{s}+\operatorname{span}\left\{a d_{f}^{\bar{k}_{s}-\mu_{l_{1}}^{s}} g_{l_{1}} \cdots a d_{f}^{\bar{k}_{s}-\mu_{l_{j}}^{s}} g_{l_{j}}\right\}$.

By construction $\Delta_{k_{s}+1}^{s+1} \equiv \bar{\Delta}_{\bar{k}_{s}-1}^{s}$. If $r=0, \Delta_{k_{s+1}}^{s+1}$ is the involutive closure of $\Delta_{k_{s}-1}^{s}$. After a possible reordering of the inputs

$$
\begin{gathered}
\bar{\Delta}_{\bar{k}_{s}-1}^{s}=\Delta_{k_{s+1}-\mu_{m}^{s+1}}^{0}+\sum_{j=1}^{\mu_{m}^{s+1}} \operatorname{span}\left\{a d_{f}^{k_{s+1}-\mu_{m}^{s+1}+j} g_{1},\right. \\
\left.\ldots, a d_{f}^{k_{s+1}-\mu_{m}^{s+1}+j} g_{i_{s+1, j}}\right\} \\
:=\Delta_{k_{s+1}}^{s+1}
\end{gathered}
$$

with the new prolongation indices $\mu_{1}^{s+1}, \ldots, \mu_{m}^{s+1}$.

Phase 2. If $\mu_{m}^{s+1}>k_{s}^{s+1}$, let $t$ be the greatest index such that $\mu_{m}^{s+1}-\mu_{t}^{s+1} \neq \mu_{m}^{s}-\mu_{t}^{s}$ and set $\rho=k_{s+1}-$ $\mu_{m}^{s+1}+\left\lfloor\left(\mu_{m}^{s+1}-\mu_{t}^{s+1}\right) / 2\right\rfloor$. If such $t$ does not exist or $\mu_{m}^{s+1} \leqslant k_{s+1}$, set $\rho=0$. For any two indices $(t, l)$ such that $t<l$ check condition (c) of Proposition 1.

Case A: Condition (c) is satisfied. If $\rho \geqslant 0$ go to Step $s+2$, else set $\tilde{k}_{s+1}=k_{s+1}-\rho$. Rename $\tilde{k}_{s+1}$ as $k_{s+1}$ and go back to Phase 2 of Step $s+1$.

Case $B$ : Condition (c) is not satisfied, i.e. there exist $\gamma$ pairs $\left(l_{p}, t_{p}\right)$, s.t.

$\left[a d_{f}^{r_{l_{p}}} g_{l_{p}}, a d_{f}^{r_{t_{p}}} g_{t_{p}}\right] \in \Delta_{k_{s}}^{s}$, while

$$
\left[a d_{f}^{r_{l_{p}}} g_{l_{p}}, a d_{f}^{r_{t_{p}}} g_{t_{p}}\right] \notin \Delta_{k_{s+1}}^{s+1}, \quad \text { for } p=1, \ldots, \gamma .
$$

Let $\bar{\gamma} \geqslant \gamma$ be the smallest integer such that

$$
\begin{gathered}
\left\{\left[a d_{f}^{r_{l_{p}}} g_{l_{p}}, a d_{f}^{r_{t_{p}}} g_{t_{p}}\right], p=1, \ldots, \gamma\right\} \in \Delta_{k_{s+1}}^{s+1} \\
\quad+\operatorname{span}\left\{a d_{f}^{k_{s}-\mu_{j_{1}}^{s}} g_{i_{s, j_{1}}} \cdots a d_{f}^{k_{s}-\mu_{j_{\gamma}}^{s}} g_{i_{s, j_{\gamma}}}\right\} .
\end{gathered}
$$


Update Table 1 of $\Delta_{k_{s+1}}^{s+1}$ by adding the elements $a d_{f}^{k_{s}-\mu_{j_{1}}^{s}} g_{i_{s, j_{1}}} \cdots a d_{f}^{k_{s}-\mu_{j_{\gamma}}^{s}} g_{i_{s, j_{\gamma}}}$, and the prolongation indices accordingly. Iterate Case $\mathrm{B}$ with respect to the new prolongation indices until condition (c) of Proposition 1 is satisfied.

Let $\tilde{\mu}_{1}^{s+1} \cdots \tilde{\mu}_{m}^{s+1}$ be the new prolongation indices, and

$$
\begin{aligned}
\tilde{U}_{\tilde{k}_{s+1}}^{s+1}= & \Delta_{k_{s+1}}^{s+1} \\
& +\operatorname{span}\left\{a d_{f}^{k_{s}-\mu_{j_{1}}^{s}} g_{i_{s, j_{1}}} \cdots a d_{f}^{k_{s}-\mu_{j_{\gamma_{s+1}}}^{s}} g_{i_{s, j_{s+1}}}\right\} .
\end{aligned}
$$

Let $r$ be the first index such that

$a d_{f}^{r} a d_{f}^{k_{s}-\mu_{j p}^{s}} g_{i_{s, j p}} \in \Delta_{k_{s+1}+r}^{s+1}, \quad p \in\left[1, \gamma_{s+1}\right]$.

Set $\hat{k}_{s+1}=\tilde{k}_{s+1}+r$, rename $\hat{k}_{s+1}$ as $k_{s+1}$ and go back to Phase 2, Step $s+1$.

Step $k^{*}$ : Suppose that the algorithm ends with prolongation indices $0=\mu_{1}^{k^{*}} \leqslant \cdots \leqslant \mu_{m}^{k^{*}}$. Consider the extended system obtained by adding the dynamic compensator (2) with $\mu_{i}=\mu_{i}^{k^{*}}, i=1, \ldots, m$. If $\mu_{m} \leqslant 2$ stop else apply the algorithm on the new system.

Let us note that at Step $s+1$-Phase 1, the number $j$ of elements added to $\Delta_{k_{s}-1}$ is in general greater than $p_{s+1}=\operatorname{dim} \bar{\Delta}_{k_{s}-1}-\operatorname{dim} \Delta_{k_{s}-1}$; moreover the choice of the elements to be added may not be unique though the number of possible choices is finite. This problem can be overcome if a more general compensator is considered, which uses static state feedback also. In this case a recombination of the input channels permits to get the $p_{s+1}$ desired directions. As for Phase 2, note that condition (c) of Proposition 1 is trivially satisfied whenever $\mu_{l}-\mu_{t} \leqslant 1$, which happens for example at Step 1. The flag $\rho$ is needed to determine if checking condition (c) on $\Delta_{k_{s+1}}^{s+1}$ is sufficient.

Next result, proven in the Appendix, shows that at the generic step $s+1$ the algorithm computes a partition of the inputs and prolongation indices $\left(\mu_{1}^{s+1}, \ldots, \mu_{m}^{s+1}\right)$ w.r.t. which the associated distributions $\Delta_{i}^{s+1}$ for $i \geqslant k_{s+1}$ satisfy Proposition 1. If the algorithm ends and $\mu_{m} \leqslant 2$ then these conditions are also sufficient and the dynamic controller defined by the given prolongation indices solves the problem. Else if $\mu_{m}>2$ one must iterate the procedure on the extended system. In this case the final controller may have $\mu_{1} \neq 0$.
Theorem 3. Suppose that Step $s+1$ ends with prolongation indices $\left(\mu_{1}^{s+1}, \ldots, \mu_{m}^{s+1}\right)$. Then $\forall i \geqslant 0 \Delta_{k_{s+1}+i}^{s}$ is involutive and satisfies the conditions of Proposition 1.

Next example consists of a system dynamic feedback linearizable with prolongation indices $\mu_{1}=0$, $\mu_{2}=3$, which does not satisfy Theorem 2 [7]. We will show how the proposed algorithm computes the above prolongation indices.

Example. Consider the system

$$
\begin{aligned}
& \dot{x}_{1}=x_{2}+x_{3} u_{2}, \quad \dot{x}_{2}=x_{3}+x_{1} u_{2}, \\
& \dot{x}_{3}=u_{1}+x_{2} u_{2}, \quad \dot{x}_{4}=u_{2} .
\end{aligned}
$$

Step 0: Set $\mu_{1}^{0}=\mu_{2}^{0}=0$ and compute the distributions $\Delta_{0}^{0}, \ldots, \Delta_{3}^{0}$ which are

$$
\begin{aligned}
& \Delta_{0}^{0}=\operatorname{span}\left\{\frac{\partial}{\partial x_{3}}, x_{3} \frac{\partial}{\partial x_{1}}+x_{1} \frac{\partial}{\partial x_{2}}+x_{2} \frac{\partial}{\partial x_{3}}+\frac{\partial}{\partial x_{4}}\right\}, \\
& \Delta_{1}^{0}=\Delta_{0}^{0}+\operatorname{span}\left\{\frac{\partial}{\partial x_{2}},-x_{1} \frac{\partial}{\partial x_{1}}+\frac{\partial}{\partial x_{3}} x_{3}\right\}, \\
& \Delta_{2}^{0}=\Delta_{3}^{0} \equiv \mathbb{R}^{4} .
\end{aligned}
$$

Locally around the origin $\operatorname{dim}\left(\Delta_{2}^{0}\right)=4$, while $\operatorname{dim}\left(\Delta_{1}^{0}\right)=3$ in the origin and $\operatorname{dim}\left(\Delta_{1}^{0}(x)\right)=4$ as soon as $x_{1} \neq 0$, i.e. the origin is a singular point for $\Delta_{1}^{0}$.

Step 1: [Phase 1] $\left[g_{1}, g_{2}\right]=a d_{f}^{2} g_{1}$, so that $\Delta_{1}^{0}$ is not involutive and we must compute $\bar{\Delta}_{1}^{0}$, which is given by

$$
\begin{aligned}
\bar{\Delta}_{1}^{0} & =\Delta_{1}^{0}+\operatorname{span}\left\{\frac{\partial}{\partial x_{1}}\right\} \\
:=\Delta_{2}^{1} & \begin{array}{|l||l|l|l|}
\hline & g_{1} & a d_{f} g_{1} & a d_{f}^{2} g_{1} \\
\hline \frac{\partial}{\partial \zeta_{21}} & g_{2} & a d_{f} g_{2} & \\
\hline
\end{array} \quad \text { with }\left\{\begin{array}{l}
\mu_{1}^{1}=0, \\
\mu_{2}^{1}=1 .
\end{array}\right.
\end{aligned}
$$

Step 2: [Phase 1] Consider now $\Delta_{1}^{1}=\left\{g_{1}, g_{2}, a d_{f} g_{1}\right\}$. One thus gets that

$$
\begin{aligned}
& \bar{\Delta}_{1}^{1}=\Delta_{1}^{1}+\operatorname{span}\left\{\frac{\partial}{\partial x_{1}}\right\}
\end{aligned}
$$

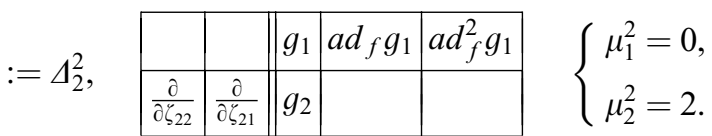


[Phase 2] $\rho=1>0$. We must now check condition (c) of Proposition 1, i.e. if $\left[a d_{f} g_{2}, g_{1}\right] \in \Delta_{2}^{2}$. Since $\left[a_{f} g_{2}, g_{1}\right]=g_{1} \in \Delta_{2}^{2}$, we can go to the next step.

Step 3: [Phase 1] Compute $\Delta_{1}^{2}=\left\{g_{1}, a d_{f} g_{1}\right\}$ and its involutive closure

$$
\begin{aligned}
& \bar{\Delta}_{1}^{2} \equiv \Delta_{1}^{2}=\operatorname{span}\left\{\frac{\partial}{\partial x_{3}}, \frac{\partial}{\partial x_{2}}\right\} \\
&:=\Delta_{1}^{3}, \\
& \begin{array}{|l|l||l|l|}
\hline & & g_{1} & a d_{f} g_{1} \\
\hline \partial \zeta_{22} & \frac{\partial}{\partial \zeta_{21}} & & \\
\hline
\end{array} \quad\left\{\begin{array}{l}
\mu_{1}^{3}=0, \\
\mu_{2}^{3}=2 .
\end{array}\right.
\end{aligned}
$$

[Phase 2] $\rho=0$. In this case condition (c) of Proposition 1 is not satisfied, since $\left[g_{2}, g_{1}\right] \notin \Delta_{1}^{3}$ whereas $\left[g_{2}, g_{1}\right] \in \Delta_{1}^{3}+\operatorname{span}\left\{a d_{f}^{2} g_{1}\right\}$. Set

$$
\begin{aligned}
& \tilde{\Delta}_{2}^{3}=\Delta_{1}^{3}+\operatorname{span}\left\{\frac{\partial}{\partial x_{3}}\right\}, \\
& \begin{array}{|c|c|c||c|c|c|}
\hline & & & g_{1} & a d_{f} g_{1} & a d_{f}^{2} g_{1} \\
\hline \frac{\partial}{\partial \zeta_{23}} & \frac{\partial}{\partial \zeta_{22}} & \frac{\partial}{\partial \zeta_{21}} & & &
\end{array} \quad\left\{\begin{array}{l}
\tilde{\mu}_{1}^{3}=0, \\
\tilde{\mu}_{2}^{3}=3 .
\end{array}\right.
\end{aligned}
$$

Condition (c) of Proposition 1 is satisfied for the new distribution $\tilde{\Delta}_{2}^{3}$, with the new prolongation indices. In fact $r_{1}+r_{2}=0$ and $\left[g_{2}, g_{1}\right] \in \tilde{\Delta}_{2}^{3}$.

According to the algorithm we must compute the first index $r: a d_{f}^{r}\left[g_{2}, g_{1}\right]=a d_{f}^{r+2} g_{1} \in \Delta_{1+r}^{3}$. In the present case, $r=1$ since $a d_{f}^{3} g_{1}=0$. We thus set $k_{3}=2$ and $\Delta_{2}^{3}=\tilde{\Delta}_{2}^{3}$. The new distribution $\Delta_{2}^{3}$ is characterized by $\rho=0$ and satisfies condition (c) so that we go to next step.

Step 4: [Phase 1] Compute $\Delta_{1}^{3}=\left\{g_{1}, a d_{f} g_{1}\right\}$ and its involutive closure

$$
\begin{aligned}
& \bar{\Delta}_{1}^{3}=\Delta_{1}^{3}=\operatorname{span}\left\{\frac{\partial}{\partial x_{3}},-\frac{\partial}{\partial x_{2}}\right\}
\end{aligned}
$$

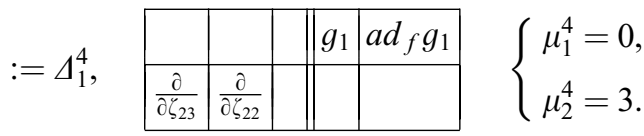

[Phase 2] $\rho=0$. Since condition (c) of Proposition 1 is trivially satisfied, we can go to next step.
Step 5: [Phase 1] compute $\Delta_{0}^{4}$ and its involutive closure which is given by

$$
\begin{aligned}
& \bar{\Delta}_{0}^{4}=\Delta_{0}^{4}=\operatorname{span}\left\{\frac{\partial}{\partial x_{3}}\right\}
\end{aligned}
$$

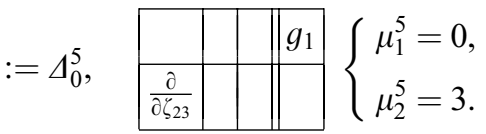

[Phase 2] $\rho=0$. Condition (c) of Proposition 1 is trivially satisfied. The algorithm ends with $\mu_{1}=\mu_{1}^{5}=0$ and $\mu_{2}=\mu_{2}^{5}=3$, which are the searched prolongation indices.

For the extended system (1)-(2) the linearizing output functions are $y_{1}=x_{4}$ and $y_{2}=x_{1}-x_{2} \zeta_{21}$, or, equivalently, the flat outputs for system (1) are $\tilde{y}_{1}=x_{4}$ and $\tilde{y}_{2}=x_{1}-x_{2} u_{2}$.

\section{Conclusions}

In this work we have proposed an algorithm for computing a dynamic controller, based on prolongations, which renders a given system linearizable via regular static feedback. The algorithm is based on geometric necessary conditions which are sufficient if the prolongation indices are at most equal to 2 .

\section{Acknowledgements}

The authors wish to thank Salvatore Monaco for suggesting and encouraging the present work.

\section{Appendix A.}

Proof of Proposition 1. Since the $\mathscr{G}_{i}^{e}$ 's satisfy the conditions of Theorem 1 they must have constant dimension around $\left(x_{0}, \zeta_{0}\right)=\left(x_{0}, 0\right)$, which implies according to (7)-(8) the necessity of (a). Condition (b) is a controllability condition and thus clearly necessary. As for (c), due to the involutivity of $\mathscr{G}_{i}^{e}$, for any $j_{1}, j_{2} \leqslant i$, and any $l, t \in[1, m],\left[a d_{F}^{j_{1}} G_{l}, a d_{F}^{j_{2}} G_{t}\right] \in \mathscr{G}_{i}^{e}$. Recall that

$a d_{F}^{j_{1}} G_{l}=(-1)^{j_{1}} \frac{\partial}{\partial \zeta_{l,\left(\mu_{l}-j_{1}\right)}}, \quad$ for $j_{1} \leqslant \mu_{l}-1$, 
$a d_{F}^{j_{2}} G_{t}=(-1)^{\mu_{t}} \tau_{t}^{\left(j_{2}-\mu_{t}\right)} \frac{\partial}{\partial x}, \quad$ for $j_{2} \geqslant \mu_{t}$.

Consider now $t<l$ and such that $i>\mu_{t}$. Then for $j_{2} \geqslant \mu_{t}$, and $0 \leqslant j_{1} \leqslant \mu_{l}-1$

$$
\begin{aligned}
& (-1)^{\left(j_{1}+\mu_{t}\right)}\left[a d_{F}^{j_{1}} G_{l}, a d_{F}^{j_{2}} G_{t}\right] \\
& =\frac{\partial\left(\tau_{t}^{\left(j_{2}-\mu_{t}\right)}(x, \zeta)\right)}{\partial \zeta_{l,\left(\mu_{l}-j_{1}\right)}} \frac{\partial}{\partial x} \in \mathscr{G}_{i}^{e} .
\end{aligned}
$$

Setting $s=j_{2}-\mu_{t}$ and $k=\mu_{l}-j_{1}$, let us note that $\frac{\partial\left(\tau_{t}^{s}(x, \zeta)\right)}{\partial \zeta_{l, k}} \frac{\partial}{\partial x}=0 \quad$ for $s<k$,

whereas for $s \geqslant k$ one gets from (7)

$$
\begin{aligned}
& \sum_{j=0}^{s-k}\left(\begin{array}{c}
k-1+j \\
k-1
\end{array}\right) a d_{f}^{j}\left[g_{l}, a d_{f}^{s-k-j} g_{t}\right] \frac{\partial}{\partial x} \\
& \quad+\gamma(x, \zeta) \frac{\partial}{\partial x} \in \mathscr{G}_{i}^{e}
\end{aligned}
$$

with $\gamma(x, 0)=0$. Since this relation must be satisfied for any $(x, \zeta)$ in a neighborhood of $\left(x_{0}, 0\right)$, it must be satisfied for $\zeta=0$. As a consequence

$\sum_{j=0}^{s-k}\left(\begin{array}{c}k-1+j \\ k-1\end{array}\right) a d_{f}^{j}\left[g_{l}, a d_{f}^{s-k-j} g_{t}\right] \in \Delta_{i}$.

Set $k_{\min }=\max \left\{\left(\mu_{l}-i\right), 1\right\}$, and fix $(s-k) \in$ $\left[\max \left\{\left(i-\mu_{l+1}\right), 0\right\}, i-\mu_{t-k}\right]$. Then for $k \in\left[k_{\min }, \mu_{l}\right]$, one gets the set of independent relations

$$
\begin{aligned}
& \left(\begin{array}{c}
k-1 \\
k-1
\end{array}\right)\left[g_{l}, a d_{f}^{s-k} g_{t}\right] \\
& +\left(\begin{array}{c}
k \\
k-1
\end{array}\right) a d_{f}\left[g_{l}, a d_{f}^{s-k-1} g_{t}\right] \\
& +\cdots+\left(\begin{array}{c}
s-1 \\
k-1
\end{array}\right) a d_{f}^{s-k}\left[g_{l}, g_{t}\right] \in \Delta_{i}
\end{aligned}
$$

in the unknowns $a d_{f}^{j}\left[g_{l}, a d_{f}^{s-k-j} g_{t}\right]$, with $j=$ $0, \ldots, s-k$. Due to the involutivity of $\Delta_{i}$, for

$0 \leqslant j \leqslant i-\mu_{l}, \quad a d_{f}^{j}\left[g_{l}, a d_{f}^{s-k-j} g_{t}\right] \in \Delta_{i}$,

which can be proven by using the Jacobi identity. For

$s-k \leqslant i-\mu_{l}+\left\lfloor\frac{\mu_{l}-\mu_{t}}{2}\right\rfloor$ one thus gets $j \leqslant \mu_{l}-k_{\min }$ independent equations in $j \leqslant \bar{j}$ unknowns. As a consequence for

$r_{1}+r_{2}=i-\mu_{l}+1, \ldots, i-\mu_{l}+\left\lfloor\frac{\mu_{l}-\mu_{t}}{2}\right\rfloor$,

each term $\left[a d_{f}^{r_{1}} g_{l}, a d_{f}^{r_{2}} g_{t}\right] \in \Delta_{i}$ even though $a d_{f}^{r_{1}} g_{l}$ does not, thus proving (c). Finally, the sufficiency of these conditions if $\mu_{m} \leqslant 2$ can be easily proven by noting that in this case (c) implies condition (iii) of Theorem 2.

Proof of Theorem 3. The proof is iterative. At step 0 , $\mu_{1}^{0}=\cdots=\mu_{m}^{0}=0$ and $\Delta_{k_{0}}^{0}$ has by assumption constant dimension $n$ locally around $x_{0}$, so that Proposition 1 is satisfied for any $\Delta_{k_{0}+i}^{0}$, with $i \geqslant 0$. Iteratively, suppose that at step $s$, for any $i \geqslant 0, \Delta_{k_{\mathrm{s}}+i}^{s}$ satisfies Proposition 1. Let the prolongation indices be $0=\mu_{1}^{s} \leqslant \cdots \leqslant \mu_{m}^{s}$ and go to Step $s+1$. We must show that the operations which modify the prolongation indices preserve properties (a) and (c) of Proposition 1 on the new distributions $\Delta_{k_{s}+i}^{s}$, for any $i \geqslant 0$.

Phase 1. Consider

$$
\begin{aligned}
\Delta_{k_{s+1}}^{s+1} & =\Delta_{\bar{k}_{s}-1}^{s}+\operatorname{span}\left\{a d_{f}^{\bar{k}_{s}-\mu_{l_{1}}^{s}} g_{l_{1}} \cdots a d_{f}^{\bar{k}_{s}-\mu_{l_{j}}^{s}} g_{l_{j}}\right\} \\
& \equiv \bar{\Delta}_{\bar{k}_{s}-1}^{s} .
\end{aligned}
$$

By construction each added element

$$
\begin{aligned}
& a d_{f}^{\bar{k}_{s}-\mu_{l_{t}}^{s}} g_{l_{t}} \in \Delta_{\bar{k}_{s}-1}^{s}+\operatorname{span}\left\{\sigma_{i}, i=1 \cdots p_{s+1}\right\}, \\
& \quad t \in[1, j],
\end{aligned}
$$

where $\sigma_{i}=\left[\tau_{1}\left[\cdots\left[\tau_{r-1}, \tau_{r}\right] \cdots\right] \cdots\right]$ and $\tau_{l} \in \Delta_{\bar{k}_{s}-1}^{S}$. By assumption $\Delta_{\bar{k}_{s}}^{s}$ is involutive $\left(\bar{k}_{s} \geqslant k_{s}\right)$, so that $a d_{f} \sigma_{i}=\sum_{j=1}^{r-1}\left[\tau_{1}\left[\cdots\left[a d_{f} \tau_{j}\left[\cdots\left[\tau_{r-1}, \tau_{r}\right] \cdots\right]\right]\right]\right] \in \Delta_{\bar{k}_{s}}^{s}$. Consequently $a d_{f}^{k_{s}-\mu_{l_{t}}^{s}+1} g_{l_{t}} \in \Delta_{\bar{k}_{s}}^{s}+\operatorname{span}\left\{\sigma_{i}, a d_{f} \sigma_{i}, i=\right.$ $\left.1, \ldots, p_{s+1}\right\} \subseteq \Delta_{\bar{k}_{s}}^{s}$, for any $t \in[1, j]$, and for any $i \geqslant 0$, $a d_{f}^{k_{s}-\mu_{l_{t}}^{s}+i+1} g_{l_{t} \in \Delta_{\bar{k}_{s}+i}^{s}} \equiv \bar{\Delta}_{\bar{k}_{s}+i}^{s}$,

which proves, $\forall i \geqslant 0$, the involutivity and constant dimensionality of $\Delta_{k_{s+1}+i}^{s+1}$, since

$$
\begin{aligned}
\Delta_{k_{s+1}+i}^{s+1}= & \Delta_{\overline{k_{s}}-1+i}^{s} \\
& +\operatorname{span}\left\{a d_{f}^{\bar{k}_{s}-\mu_{l_{1}}^{s}+i} g_{l_{1}} \cdots a d_{f}^{\overline{k_{s}}-\mu_{l_{j}}^{s}+i} g_{l_{j}}\right\} \\
\equiv & \bar{\Delta}_{\overline{k_{s}}-1+i}^{s} .
\end{aligned}
$$


Phase 2. According to the algorithm, three situations are possible. Case A: Condition (c) is satisfied for $\Delta_{k_{s+1}}^{s+1}$ and $\rho \geqslant 0$. Iteratively, suppose it satisfied for $\Delta_{j}^{s+1}, j \geqslant k_{s+1}$. Assume first

$j-\mu_{l}^{s+1}+\left\lfloor\frac{\mu_{l}^{s+1}-\mu_{t}^{s+1}}{2}\right\rfloor \geqslant 0$.

We must verify that

$$
\begin{aligned}
\forall \bar{r}_{1}, \bar{r}_{2} \geqslant 0: \quad \bar{r}_{1}+\bar{r}_{2}= & j-\mu_{l}^{s+1}+2, \ldots, j-\mu_{l}^{s+1}+1 \\
& +\left\lfloor\frac{\mu_{l}^{s+1}-\mu_{t}^{s+1}}{2}\right\rfloor
\end{aligned}
$$

$\left[a d_{f}^{\bar{r}_{1}} g_{t}, a d_{f}^{\bar{r}_{2}} g_{l}\right] \in \Delta_{j+1}^{s+1}$.

It is sufficient to check that the previous relation is satisfied if $\mu_{l}^{s+1}-\mu_{t}^{s+1}>1$, and since

$\Delta_{j+1}^{s+1} \supset \Delta_{j}^{s+1}$

$$
\text { for } \bar{r}_{1}+\bar{r}_{2}=j-\mu_{l}^{s+1}+1+\left\lfloor\frac{\mu_{l}^{s+1}-\mu_{t}^{s+1}}{2}\right\rfloor,
$$$$
\bar{r}_{2} \geqslant j-\mu_{l}^{s+1}+2 .
$$

Set $\bar{r}_{2}=j-\mu_{l}^{s+1}+2$, consequently

$\bar{r}_{1}=\left\lfloor\frac{\mu_{l}^{s+1}-\mu_{t}^{s+1}}{2}\right\rfloor-1$.

Recall that for

$0 \leqslant i \leqslant\left\lfloor\frac{\mu_{l}^{s+1}-\mu_{t}^{s+1}}{2}\right\rfloor,\left[a d_{f}^{\bar{r}_{1}-i} g_{t}, a d_{f}^{\bar{r}_{2}+i-1} g_{l}\right] \in \Delta_{j}^{s+1}$,

so that by construction

$$
\begin{aligned}
\Delta_{j+1}^{s+1} \ni a d_{f}\left[a d_{f}^{\overline{r_{1}}-i} g_{t}, a d_{f}^{\overline{r_{2}}+i-1} g_{l}\right] \\
=\left[a d_{f}^{\overline{r_{1}}-i+1} g_{t}, a d_{f}^{\bar{r}_{2}+i-1} g_{l}\right] \\
+\left[a d_{f}^{\overline{r_{1}}-i} g_{t}, a d_{f}^{\bar{r}_{2}+i} g_{l}\right] .
\end{aligned}
$$

Iteratively, for $i=0, \ldots,\left\lfloor\mu_{l}^{s+1}-\mu_{t}^{s+1} / 2\right\rfloor$, assume that $\left[a d_{f}^{\overline{r_{1}}-i+1} g_{t}, a d_{f}^{\bar{r}_{2}+i-1} g_{l}\right] \in \Delta_{k_{s+1}+1}^{s+1}$

(true for $i=0)$ then due to (11),

$\left[a d_{f}^{\overline{r_{1}}-i} g_{t}, a d_{f}^{\overline{r_{2}}+i} g_{l}\right] \in \Delta_{k_{s+1}+1}^{s+1}$.
Assume now that for some $(t, l)$, with

$t<l, k_{s+1}-\mu_{l}^{s+1}+\left\lfloor\frac{\mu_{l}^{s+1}-\mu_{t}^{s+1}}{2}\right\rfloor<0$,

then we will first show that necessarily $\mu_{l}^{s+1}-\mu_{t}^{s+1}=$ $\mu_{l}^{s}-\mu_{t}^{s}$. In fact by assumption $\rho \geqslant 0$ so that $\mu_{m}^{s+1}-$ $\mu_{t}^{s+1}=\mu_{m}^{s}-\mu_{t}^{s}$. Moreover $\mu_{m}^{s+1} \geqslant \mu_{l}^{s+1}>k_{s+1} \geqslant 0$, so that $\mu_{m}^{s+1}-\mu_{l}^{s+1}=\mu_{m}^{s}-\mu_{l}^{s}$ and consequently $\mu_{t}^{s+1}$ $\mu_{l}^{s+1}=\mu_{t}^{s}-\mu_{l}^{s}$ and

$\mu_{l}^{s+1}-k_{s+1} \geqslant 1$.

Consider now $\Delta_{j}, j \geqslant k_{s+1}$. For

$j<\mu_{l}^{s+1}-\left\lfloor\frac{\mu_{l}^{s+1}-\mu_{t}^{s+1}}{2}\right\rfloor=\bar{j}$,

condition (c) is trivially satisfied. According to the above discussion, it is then sufficient to verify (10)

$\forall \bar{r}_{1}, \bar{r}_{2} \geqslant 0: \bar{r}_{1}+\bar{r}_{2}=1-\left\lfloor\frac{\mu_{l}^{s+1}-\mu_{t}^{s+1}}{2}\right\rfloor, \ldots, 0$,

i.e. $\left[g_{l}, g_{t}\right] \in \Delta_{\bar{j}}^{s+1}$ for

$1-\left\lfloor\frac{\mu_{l}^{s+1}-\mu_{t}^{s+1}}{2}\right\rfloor<0$.

Recall that $\mu_{l}^{s+1}-k_{s+1}=\mu_{l}^{s}-\bar{k}_{s}+1$. Moreover, due to (9), $\Delta_{\bar{j}}^{s+1} \equiv \Delta_{\bar{k}_{s}+\bar{j}-k_{s+1}-1}^{s}$, and by assumption $\Delta_{\bar{k}_{s}+i-1}^{s}$ verifies Proposition 1 , for any $i \geqslant 0$, so that $\left[g_{l}, g_{t}\right] \in \Delta_{\bar{k}_{s}+\bar{j}-k_{s+1}-1}^{s} \equiv \Delta_{\bar{j}}^{s+1}$, which ends the proof since $\bar{k}_{s}-k_{s+1}-\mu_{l}^{s}+\bar{j}<0$ whereas

$\bar{k}_{s}-k_{s+1}-\mu_{l}^{s}+\bar{j}-1+\left\lfloor\frac{\mu_{l}^{s}-\mu_{t}^{s}}{2}\right\rfloor=0$.

Case A: Condition (c) is satisfied but $\rho<0$. In this case $\mu_{m}^{s+1}-\mu_{t}^{s+1} \neq \mu_{m}^{s}-\mu_{t}^{s}$, and checking condition (c) on $\Delta_{k_{s+1}}^{s+1}$ does not guarantee that it is satisfied also by $\Delta_{k_{s+1}+i}^{s+1}$, for any $i \geqslant 0$. In order to verify condition (c), the algorithm must check it on $\Delta_{k_{s+1}-\rho}^{s+1}$ which is already involutive. It thus sets $\tilde{k}_{s+1}=k_{s+1}-\rho$, renames $\tilde{k}_{s+1}$ as $k_{s+1}$ and goes back to Phase 2 of Step $s+1$.

Case B: Condition (c) is not satisfied, i.e. there exist $\gamma$ pairs of indices $\left(l_{p}, t_{p}\right)$, s.t.

$\left[a d_{f}^{r_{l_{p}}} g_{l_{p}}, a d_{f}^{r_{t_{p}}} g_{t_{p}}\right] \in \Delta_{k_{s}}^{s}$,

while $\left[a d_{f}^{r_{l_{p}}} g_{l_{p}}, a d_{f}^{r_{t_{p}}} g_{t_{p}}\right] \notin \Delta_{k_{s+1}}^{s+1}$,

for $p=1, \ldots, \gamma$. 
The algorithm computes the smallest set of elements such that

$$
\begin{gathered}
\left\{\left[a d_{f}^{r_{l_{p}}} g_{l_{p}}, a d_{f}^{r_{t_{p}}} g_{t_{p}}\right], p=1, \ldots, \gamma\right\} \in \Delta_{k_{s+1}}^{s+1} \\
\quad+\operatorname{span}\left\{a d_{f}^{k_{s}-\mu_{j_{1}}^{s}} g_{i_{s, j_{1}}} \cdots a d_{f}^{k_{s}-\mu_{j_{\gamma}}^{s}} g_{i_{s, j_{\gamma}}}\right\} .
\end{gathered}
$$

Table 1 of $\Delta_{k_{s+1}}^{s+1}$ is updated by adding the new elements, as well as the prolongation indices. This operation is iterated until condition (c) is satisfied with respect to the new prolongation indices, $\tilde{\mu}_{1}^{s+1} \cdots \tilde{\mu}_{m}^{s+1}$.

Since involutivity is not guaranteed anymore for the new distribution

$\tilde{\Delta}_{\tilde{k}_{s+1}}^{s+1}=\Delta_{k_{s+1}}^{s+1}+\operatorname{span}\left\{a d_{f}^{k_{s}-\mu_{j_{1}}^{s}} g_{i_{s, j_{1}}} \cdots a d_{f}^{k_{s}-\mu_{j_{s+1}}^{s}} g_{i_{s, j_{\gamma_{s}+1}}}\right\}$,

the algorithm computes the smallest integer $r$ such that $\widetilde{\Delta}_{\tilde{k}_{s+1}+r}^{s+1} \equiv \Delta_{k_{s+1}+r}^{s+1}$. The algorithm sets $\hat{k}_{s+1}=k_{s+1}$ $+r$, renames $\hat{k}_{s+1}$ as $k_{s+1}$ and goes back to Phase 2, since the new added vector fields change the prolongation indices but not the involutivity and constant dimensionality properties of $\Delta_{k_{s+1}+r}^{s+1}$ proved above, nor those of $\Delta_{k_{s+1}+r+i}^{s+1}$ for any $i \geqslant 0$.

\section{References}

[1] E. Aranda-Bricaire, U. Kotta, C.H. Moog, Linearization of discrete-time systems, SIAM J. Control Optim. 34 (1996) 1999-2023.

[2] E. Aranda-Bricaire, C.H. Moog, J.-B. Pomet, A linear algebraic framework for dynamic feedback linearization, IEEE Trans. Automat. Control 40 (1995) 127-132.

[3] S. Battilotti, C. Califano, Further results on dynamic feedback linearization, Proceedings of ECC03, Cambridge, UK, 2003.

[4] R.W. Brockett, Feedback invariants for nonlinear systems, IFAC, 1978, pp. 1115-1120.

[5] C. Califano, S. Monaco, D. Normand-Cyrot, On the feedback linearization problem, Systems Control Lett. 36 (1999) $61-67$.
[6] B. Charlet, J. Lévine, R. Marino, On dynamic feedback linearization, Systems Control Lett. (1989) 143-151.

[7] B. Charlet, J. Lévine, R. Marino, Sufficient conditions for dynamic feedback linearization, SIAM J. Control Optim. 29 (1991) 38-57.

[8] M. Fliess, J. Lévine, P. Martin, P. Rouchon, Flatness and defect of nonlinear systems: introductory theory and examples, Internat. J. Control 61 (1995) 1327-1361.

[9] M. Fliess, J. Lévine, P. Martin, P. Rouchon, A Lie-Backlund approach to equivalence and flatness of nonlinear systems, IEEE Trans. Automat. Control 44 (1999) 922-937.

[10] L.R. Hunt, R. Su, G. Meyer, Design for multi-input nonlinear systems, in: R.W. Brockett, R.S. Millman, H. Sussmann (Eds.), Differential Geometric Control Theory, Birkhauser, Basel, 1983, pp. 268-298.

[11] A. Isidori, Nonlinear Control Systems: An Introduction, 3rd Edition, Springer, Berlin, 1995.

[12] A. Isidori, A.J. Krener, C. Gori Giorgi, S. Monaco, Decoupling via feedback: a differential geometric approach, IEEE Trans. Automat. Control 26 (1981) 331-345.

[13] A. Isidori, C.H. Moog, A. de Luca, A sufficient condition for full linearization via dynamic state feedback, Proceedings of the 25th IEEE CDC, New York, USA, 1986, pp. 203-208.

[14] B. Jakubczyk, Feedback linearization of discrete-time systems, Systems Control Lett. 9 (1987) 441-446.

[15] B. Jakubczyk, W. Respondek, On linearization of control systems, Bull. Acad. Polonaise Sci. 28 (1980) 517-522.

[16] H.G. Lee, A. Arapostathis, S.I. Marcus, On the linearization of discrete-time systems, Internat. J. Control 45 (1987) $1783-1785$

[17] R. Marino, On the largest feedback linearizable subsystem, Systems Control Lett. 6 (1986) 345-351.

[18] S. Monaco, D. Normand-Cyrot, On the immersion under feedback of a multidimensional discrete time nonlinear system into a linear system, Internat. J. Control 38 (1983) 245-261.

[19] S. Monaco, D. Normand-Cyrot, Minimum phase nonlinear discrete-time systems and feedback stabilization, Proceedings of the 26th IEEE CDC, New York, USA, 1987, pp. 979-986.

[20] H. Nijmeijer, A.J. Van der Schaft, Nonlinear Dynamical Control Systems, Springer, Berlin, 1990.

[21] W.M. Sluis, D.M. Tilbury, A bound on the number of integrators needed to linearize a control system, Systems Control Lett. 29 (1996) 43-50. 